\title{
Une presse à huile au Maroc
}

A twin-screw oil press from Morocco

\section{Narjys El alaoui}

\section{OpenEdition}

\section{Journals}

Édition électronique

URL : https://journals.openedition.org/tc/4541

DOI : $10.4000 /$ tc. 4541

ISSN : 1952-420X

\section{Éditeur}

Éditions de l'EHESS

\section{Édition imprimée}

Date de publication : 12 décembre 2007

Pagination : 189-218

ISSN : 0248-6016

\section{Référence électronique}

Narjys El alaoui, « Une presse à huile au Maroc », Techniques \& Culture [En ligne], 48-49 | 2007, mis en ligne le 20 juin 2010, consulté le 29 septembre 2022. URL : http://journals.openedition.org/tc/4541 ; DOI : https://doi.org/10.4000/tc.4541 


\section{Narjys El Alaoui*}

\section{Une presse à huile au Maroc}

Cette note décrit la presse à huile en bois à vis latérales du Prérif (Maroc du Nord) et sinterroge sur les conditions de son maintien à la lumière de son contexte d'utilisation et d'autres presses et procédés d'extraction de l'huile d'olive. Le traitement caractéristique de l'olive, depuis sa cueillette jusqu'à l'extraction d'une huile appelée 'alwana et le procédé exclusif aux femmes retiendra particulièrement l'attention. Laspect lacunaire des sources écrites sur cette presse, vraisemblablement connue depuis le $I^{\text {er }}$ siècle $A D$ et son remplacement récent par une presse métallique montrent que la réalité du patrimoine est imputable au scribeobservateur des faits (techniques) et non à l'existence seule de l'objet, de l'outil ou de la technique qui peuvent disparaître sans laisser de trace.

Maroc, Taounate, huile d’olive, presse en bois à vis latérales, procédé technique féminin, ‘lwana.

Succédant à de brèves missions dans les régions oléicoles du Maroc (Souss en 200I et Prérif en 2002, 2003) ${ }^{1}$, cette note cherche à mettre en lumière la présence et le fonctionnement d'un type particulier de presse à huile sur lequel nous n'avions aucun témoignage ni description dans le contexte de son utilisation en Méditerranée. Il s’agit d’une presse en bois à deux vis latérales, actuellement utilisées au Maroc sous deux formats différents et pour deux usages distincts, dans la province de Taounate où la géographie, le climat et l'écart des voies de circulation ont probablement commandé son maintien.

Située dans la région Centre-Nord du Maroc, Taounate est limitée au nord par les provinces d'Al-Hoceima et de Chefchaouen, au sud par la Wilaya

\footnotetext{
*Ethnologue. Musée des Civilisations de l'Europe et de la Méditerranée. narjys.elalaoui@culture.gouv.fr. Un grand merci à MM. François Sigaut, Robert Cresswell, Jacques Vignet-Zunz, Georges Comet, Didier Gazagnadou pour les remarques et les commentaires amicaux que leur a suggéré la lecture de ce texte. Toutes les photos sont de l'auteur.

${ }^{1}$ Missions effectuées pour le musée national des Arts et Traditions populaires - CNRS (UMR 306) : novembredécembre 2002 (Souss et Taounate) et décembre 2003 (Taounate).
} 
de Fès, à l'est par la province de Taza et à l'ouest par celle de Sidi Qacem. La région est fortement peuplée (92\%) de ruraux Senhaja, Jbala et Branès arabophones du Prérif, qui occupent les collines et les plaines entre $650 \mathrm{~m}$ et I $000 \mathrm{~m}$ d'altitude et dont le mode de vie, lié à la terre, est soumis aux aléas du climat continental avec des étés chauds et secs $\left(40^{\circ} \mathrm{C}\right)$, des hivers froids $\left(5^{\circ} \mathrm{C}\right)$ et des précipitations atmosphériques d'environ $\mathrm{I} 000 \mathrm{~mm}$ par an.

Cette région se révèle d'une richesse technique peu commune. La noria, ou moulin hydraulique à palettes, mue par le courant de l'oued Sbou (au sud) et de l'oued Ourgha (au nord) reflète la fertilité des berges et l'agriculture environnante : céréaliculture (blés dur et tendre, orge en montagne), culture annuelle des légumineuses (lentille, fève, haricot blanc), maraîchage (tomate, poivron, oignon, etc.), arboriculture (forêt : chêne, pins, noyer, peuplier, mûrier; plantations fruitières: olivier [dominant $\left.{ }^{2}\right]$, figuier, grenadier, agrumes), associés à un élevage semi intensif (vache, mouton) et domestique (vache, mouton, volaille). On y rencontre la maison en pisé à cour centrale et toiture plate ou inclinée à double pente, le moulin à bras en pierre, l'araire, les moulins et les presses à huile, les silos à grains, à paille ou à olives, le puits à poulie, le mode de transport animal (âne), ou de labours (mule, âne) et la poterie domestique féminine d'argile rouge moulée à fond plat, proposée l'été sur le marché de Humis Houara.

Adapté à une économie rurale et familiale, l’usage régional et domestique des presses en bois à vis latérales répondrait à lautarcie séculaire de ces populations $^{3}$, miroir de leurs choix économique et technique. Bien que la presse à levier, vis et contrepoids ${ }^{4}$ se maintienne dans le Zerhoun (Moulay Idriss), sa disparition semble néanmoins imminente. En revanche, l'extraction de la pierre meulière, destinée au moulin à huile familial ou de coopérative, se poursuit non loin de là, à Taza (Oued Amlil) mais aussi dans la région méridionale du Souss intérieur (Tiyout).

Dans cette zone oléicole où l'énergie électrique tend à remplacer celle de l'homme et de l'animal, on remarque que diverses machines (El Alaoui 2003) se côtoient, sans se concurrencer. À l'ancien type de machines en bois, des

\footnotetext{
2 Les plantations oléicoles couvrent $23 \%$ de la surface agricole utilisable (irriguée et non irriguée).

3 Les presses métalliques à vis latérale (unique ou double) sont partout présentes dans les régions oléicoles du Maroc.

${ }^{4}$ Selon les techniciens du Centre de Travaux Agricoles - CTA de Taounate, les presses métalliques à double vis auraient fait leur apparition dans les années 1975-1980.
} 
presses métalliques à vis plus résistantes sont substituées qui révèlent un certain dynamisme pour améliorer la productivité et la qualité de l'huile.

Le regain mondial pour l'huile d'olive, la proximité de la Méditerranée et la petite taille de la presse domestique que je vais décrire, ont contribué à l'acquisition récente de celle-ci par quelques musées privés du Maroc (Nejjarine à Fès, Dar Si Saïd à Marrakech) ou nationaux (musée national des Arts et Traditions populaires à Paris, en 2003) ${ }^{5}$. Elle enrichira sans nul doute la typologie des presses à huile de Méditerranée.

\section{De la presse alexandrine...}

Sur linvention et la diffusion des presses à huile en bois à double vis en Méditerranée, nous sommes peu renseignés. Néanmoins, dans Les Mécaniques ou l'éleveur des corps lourds, Héron, ingénieur grec de l'école d'Alexandrie, nous a laissé une description fort instructive, datée du I $^{\text {er }}$ siècle $\mathrm{AD}^{6}$ d'une presse en bois pour les olives, puissante, facilement transportable et fonctionnant au moyen de deux vis ${ }^{7}$. Le texte grec étant perdu, nous disposons de la version arabe du philosophe Qustāa ibn Lūqā de Baâlbek (fin du $\mathrm{X}^{\mathrm{e}}$ siècle) qui a été rapportée d'Orient par Golius et transmise à la bibliothèque de Leyde $^{8}$. Ce texte arabe, édité et traduit en français par Bernard Carra de Vaux à la fin du XIX ${ }^{\mathrm{e}}$ siècle (Carra de Vaux I893, réédité en 1988), nous est précieux car, outre son ancienneté, il permet de comparer le vocabulaire technique arabe du $\mathrm{X}^{\mathrm{e}}$ siècle au vocabulaire actuel, tel que je l'ai recueilli dans la région de Taounate $^{9}$, où j’ai vu fonctionner la presse récemment. Fait important : les figures proposées par Carra de Vaux, Nix ou Parharides et Vallianos montrent la difficulté de représenter graphiquement la presse antique d'après la seule lecture des manuscrits, et posent le problème difficile de linterprétation. Par ailleurs, un certain nombre de détails techniques appellent l'attention tels l'écrou rond à la base de la partie

\footnotetext{
${ }^{5}$ Actuellement musée des Civilisations de l'Europe et de la Méditerranée (MuCEM).

${ }^{6}$ Date discutée, cf. Parrain $1979: 284$.

${ }^{7} \mathrm{Cf}$. Annexe.

${ }^{8}$ Réf. DCCCCC XXXIII, Cod 51 (1) GoI. D’autres manuscrits, qu'il conviendrait de consulter, ont été utilisés (cf. préface de Donald R. Hill, édition 1988 : 19)

${ }^{9}$ Cf. glossaire en annexe 1.
} 
équarrie de la vis, le nombre et la longueur de barres de rotation, la rotation de la vis semblable à celle d'une presse à levier.

En revanche, lobservation de la presse de Taounate, dans son contexte d'usage et d'utilisation facilite l'intelligence de cette description en même temps qu'elle souligne la proximité technique avec la presse antique.

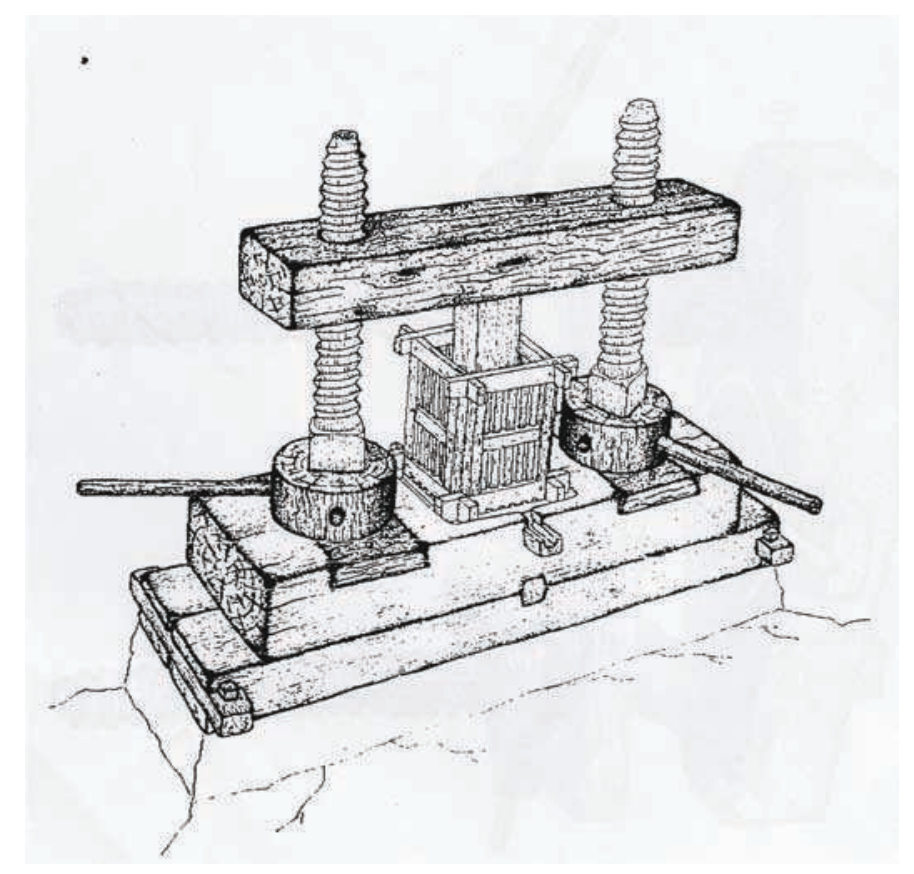

Figure 1. Presse de Héron (d'après Parharides, Ioannou \& Vallianos 2001).

\section{...̀̀ la presse de Taounate}

Linvention, voire le transfert, du panier en fibre animale ou végétale dans le pressurage de l'huile (d'olive) a marqué une étape fondamentale dans le perfectionnement de la presse antique, en éliminant l'ensemble rigide galéagre-tambour-chapeau (cf. description en annexe 2) en bois, devenu obsolète et qui jouait un rôle similaire dans le pressurage. Alors que Les Mécaniques n'apportent pas d’indication technique quant au traitement de l'olive avant sa transformation dans la presse antique (passage du solide au liquide), on admettra que l'adjonction des paniers a sans nul doute 
perfectionné la presse en libérant l'énergie et l'attention humaines. Le serrage des écrous, qui transfert la force humaine au plateau, va directement agir sur les paniers contenant la pâte d'olive, t'am ${ }^{10}$ et extraire l'huile. Ainsi, la mécanique fonctionne jusqu’à épuisement de la pâte oléagineuse sans intervention humaine continue. C'est là en effet que réside le progrès qui libère des contraintes de la presse à coins (déplacer, soulever, poser les pierres lourdes l'une sur l'autre sur un plan incliné), utilisée à Taounate.

Même si l'histoire des techniques est peu explicite quant à la qualité des olives, au type de cueillette et au processus de broyage, qui ont une incidence directe et déterminante sur l'extraction et la qualité de l'huile, on considérera lintroduction du panier souple dans la presse à vis latérales de Taounate comme le témoin discret d'un progrès technique considérable. On peut, de même, émettre l'hypothèse que la presse à vis latérales, qui induit une pression puissante et uniforme de la pâte d'olive est apparue pour améliorer les presse « à coins » et à levier encore utilisées dans la région.

Si l'on considère, par ailleurs, le rôle important de limprimerie à Fès ${ }^{11}$, on serait tenté de corréler la presse à livres (double vis) avec la presse à huile antique améliorée de Taounate. En revanche, dans le Souss (Taroudannt), région oléicole méridionale, où l'imprimerie n’a pas connu le même essor, la presse à double vis en bois est inconnue ${ }^{12}$ tandis que la presse à levier en bois et contrepoids en pierre, d'utilisation si pénible, voire dangereuse, poursuit son activité dans l'espace rural et jusqu'aux portes de la ville, mais elle cède néanmoins de plus en plus la place à la presse hydraulique à vis (une ou deux), depuis la sécheresse des années I960-I970. Le mécanisme de la presse à vis latérales ne présente pas le danger de la presse à levier (El Alaoui 200I) utilisée dans le Sud (Souss) et le Nord (Zerhoun) où la barre de rotation, engagée dans la partie circulaire du montant, durant l'extraction de l'huile, peut revenir brutalement sur louvrier distrait et le blesser grièvement. Pour immobiliser cette barre, une cavité a d'ailleurs été aménagée dans le mur de certaines huileries du Souss.

\footnotetext{
10 Lit. nourriture.

11 Au Musée Belghazi de Fès, une presse à livres (sans maie) de petite dimension avait attiré mon attention par sa ressemblance, frappante, avec la presse à huile domestique de Taounate. Au XII siècle, Fès faisait tourner des centaines de moulins à papier.

12 Le pressoir métallique à double vis domine dans les huileries semi industrielles.
} 


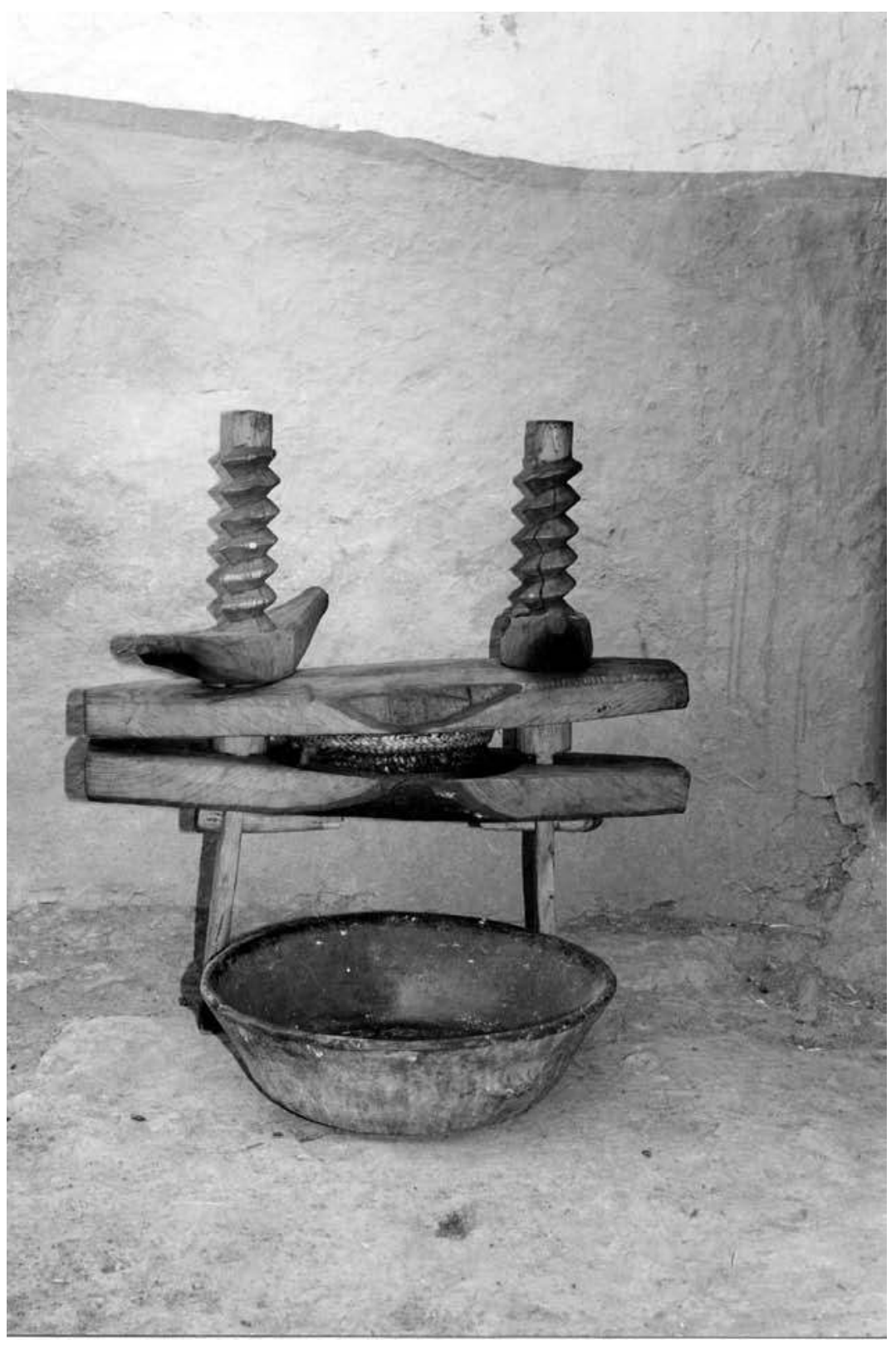

Figure 2. La presse de Taounate. 
Dans le Prérif où les femmes et les hommes apprennent très jeunes à construire leur autonomie, les qualités et l'efficacité inégalées de la presse à deux vis latérales, utilisée dans certains villages accessibles par pistes, répondent à une indépendance séculaire dont on retiendra les faits prégnants suivants : la coïncidence de la cueillette des olives avec la saison des pluies qui rendent les pistes impraticables voire inaccessibles, la disponibilité de la matière végétale, le coût relativement faible du bois et de la main d'œuvre, le savoir technique artisanal pérenne, la résistance du bois dense aux pressions du serrage en regard du rendement attendu, la possibilité de remplacer sur place chacun de ses éléments, l'entretien aisé, l’encombrement réduit, la facilité de manipulation, l'éloignement du marché et enfin l'extraction rapide d’une huile très appréciée. Ces faits conjugués concourent à son maintien.

\section{La petite presse à vis latérales}

Diverses essences locales de bois dense concourent à la réalisation artisanale de la presse domestique à double vis de Taounate : chêne, noyer, caroubier "mâle", mûrier, micocoulier. Le noyer, qui atteint ici une hauteur exceptionnelle et le caroubier sont les essences les plus estimées. Chez les Senhaja, elle est fabriquée par un ébéniste à Bu 'Adl et à In Medyouna.

Cette presse, utilisée pour la fabrication d'une huile des prémices appelée álwana, est composée de quatre éléments doubles monoxyles (fig. 2):

- deux plateaux percés à leurs extrémités pour le passage des vis. Le plateau supérieur peut être doté d'une poignée inférieure, et le plateau inférieur comporte une maie creusée sur son pourtour et un bec verseur ;

- deux vis partiellement filetées ;

- deux écrous ;

- deux clés de blocage. 


\begin{tabular}{|c|c|c|c|c|}
\hline Éléments & $\begin{array}{c}\text { Appellation } \\
\text { vernaculaire } \\
\text { Douar 'In } \\
\text { Dahir }\end{array}$ & $\begin{array}{l}\text { Essence } \\
\text { végétale }\end{array}$ & $\begin{array}{l}\text { Appellation } \\
\text { vernaculaire } \\
\text { Douar'In } \\
\text { Ma'touf }\end{array}$ & Dimensions $(\mathrm{cm})$ \\
\hline Plateau & lhawd & tizg் / Noyer & lbașt & $69 \times(15 / 29) \times 5$ \\
\hline Plateau mobile & luha & tizg $\dot{g} a$ / Noyer & hnzira & $69 \times(13,5 / 27,5) \times 5$ \\
\hline Cuvette (maie) & mida & & mida & $21 \times 2$ \\
\hline Poignée & lyed & Caroubier & & \\
\hline $\mathrm{Bec}$ & $\operatorname{lm}$ 'gun & & $z r r a b$ & 2 \\
\hline Vis & $m \dot{g} z l$ & $\begin{array}{l}\text { tiziga / Caroubier } \\
\text { / Mûrier noir }\end{array}$ & $m \dot{g} z l$ & $61,8 \times 7,5$ \\
\hline Ecrou & quqna & & hnzira & $42 \times 9 \times 6$ \\
\hline Clé de blocage & & $\begin{array}{l}\text { Bois dense sans } \\
\text { distinction }\end{array}$ & & $17 \times 3 \times 2$ \\
\hline Panier & chamya & doum / Jonc & chamya & $20 \times 10$ \\
\hline
\end{tabular}

Tableau 1. Caractéristiques de la petite presse à vis latérales.

Préparation d' 'alwana: l'huile des prémices

Parmi les procédés féminins d'extraction d'huile d’olive, que jai observés à Taounate, le plus sophistiqué s'effectue avec la presse à double vis, à partir d'olives vertes ou tournantes, cueillies à la main, chauffées dans un four à pain chaud, puis écrasées, pétries et pressées.

Caractéristique de la région, l'alwana, fort prisée ici, est l'aboutissement d'un processus opératoire rythmé par quatre étapes consécutives à la cueillette des olives : mise au four chaud (enfournement), concassage, pétrissage et pressurage du fruit. Certaines similitudes avec le procédé millénaire d'extraction de l'huile d'argan (El Alaoui 1999) doivent retenir 
notre attention : dessiccation, concassage manuel du fruit entre deux pierres, pétrissage manuel, chauffage de la pâte et ajout d'eau sont identiques.

La traite des olives

Exclusivement féminine, la cueillette manuelle des olives (fig. 3), tsram, hlib zitun ou $h l b u^{13}$ (lit. traite, le geste étant identique à celui de la traite des animaux à lait), concerne les jeunes oliviers de petite taille, ou la partie basse d'un olivier adulte non taillé (la gaule est utilisée par les femmes et les hommes pour les fruits que la main ne peut atteindre). Ces fruits mûrs, d'une belle couleur violine, sont très appréciés pour préparer l'alwana.

Figure 3. La cueillette manuelle.

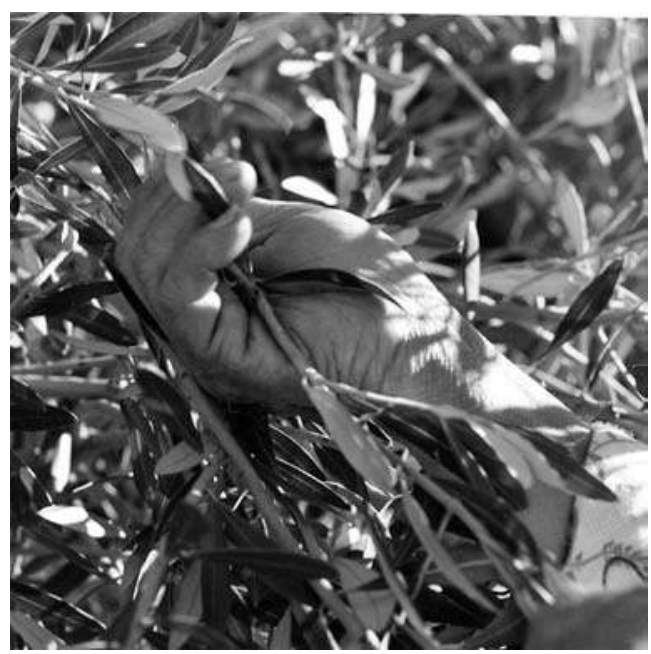

L'enfournement / la déshytratation

Dans cet environnement où la poterie à usage domestique est façonnée par les femmes, le four à pain (frna) est, de même, construit par leurs soins à l'extérieur des habitations ${ }^{14}$, à partir d'argiles rouge et grise, de sable et de rebuts de poterie concassés et tamisés pour l'assise, ou montées en colombins

\footnotetext{
13 Termes désignant la cueillette manuelle des olives ; procédé qui évite la tuberculose de l'olivier, bactérie propagée par le gaulage et les outils métalliques.

14 Chaque unité domestique dispose de deux fours construits à proximité de son habitation et dont l'utilisation est tributaire des conditions atmosphériques : l'un couvert, utilisé en hiver est caractérisé par une assise basse ; l'autre plus haut est érigé à ciel ouvert.
} 
pour les parois. Après la cuisson du pain (contrainte économique), les restes de charbons de bois et de cendres sont dégagés du four chaud, balayé.

Cinq kilogrammes d'olives fraîches (gonflées d'eau de pluie) y sont soigneusement étalés et le four fermé hermétiquement ${ }^{15}$ pendant la nuit (environ douze heures).

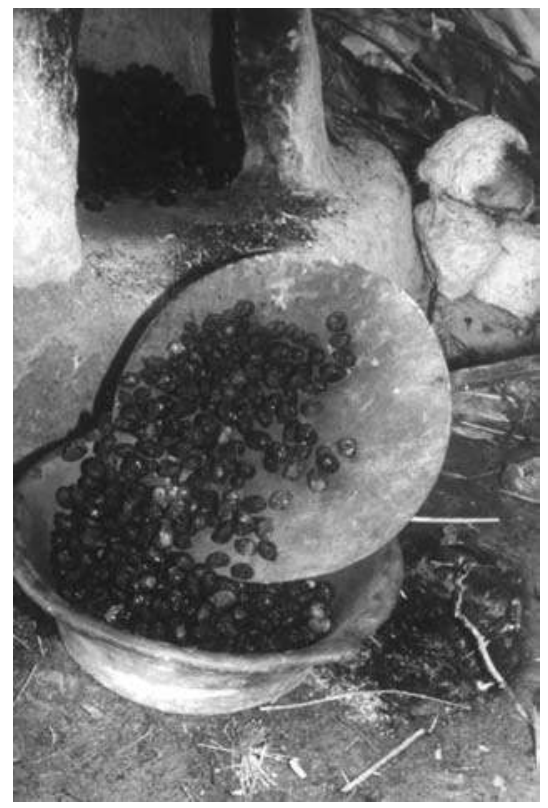

Figure 4. Olives sortant du four.

Les olives déshydratées sont alors retirées à l'aide d'une pelle (rmaya) et déposées dans une jatte en argile à deux tenons. Elles ont pris une couleur très sombre. Cette déshydratation doit être considérée comme une des étapes du processus de fabrication d'alwana car, du séchage particulier du fruit oléagineux, non signalé jusqu’ici, dans le four chaud résultera une huile dépourvue de margines (eau de végétation amère et polluante), prête à la consommation. Nécessité technique de retrait naturel des margines ${ }^{16}$, la dessiccation reflète aussi l'affect des consommateurs qui apprécient cette huile des prémices comme une " confiserie » au goût « fumé » particulier. Une technique proche avait été connue en Iran au $\mathrm{XVI}^{\mathrm{e}}$ siècle :

15 Le couvercle métallique du four est scellé à l'aide d'argile.

16 Les Idaw Martini de l'Anti-Atlas central exposent leurs olives fraîches au soleil d'hiver pour une extraction ultérieure de l'huile. 
«On laisse les olives sur l'arbre tant qu'elles ne sont pas encore noires. Ensuite, on les cueille et on les met dans un fourneau. On ferme le fourneau pour que les olives soient brûlées. Ensuite, on les retire. Sur un récipient, une planche de bois est mise. On met les olives sur cette planche et sur celle-ci on fixe une deuxième planche. Les deux planches sont liées par une corde assez fortement pour que l'huile coule dans un récipient" (Mohebbi I996 : 22I).

\section{Le concassage}

Cette opération suit immédiatement le défournement. Dans un large van spiralé (miduna), servant, selon les nécessités, de pétrin ou de tamis à la volée, deux femmes, assises l'une en face de l'autre, écrasent entre deux pierres les olives chaudes contenues dans la jatte (fig. 5).

Figure 5. Le concassage.

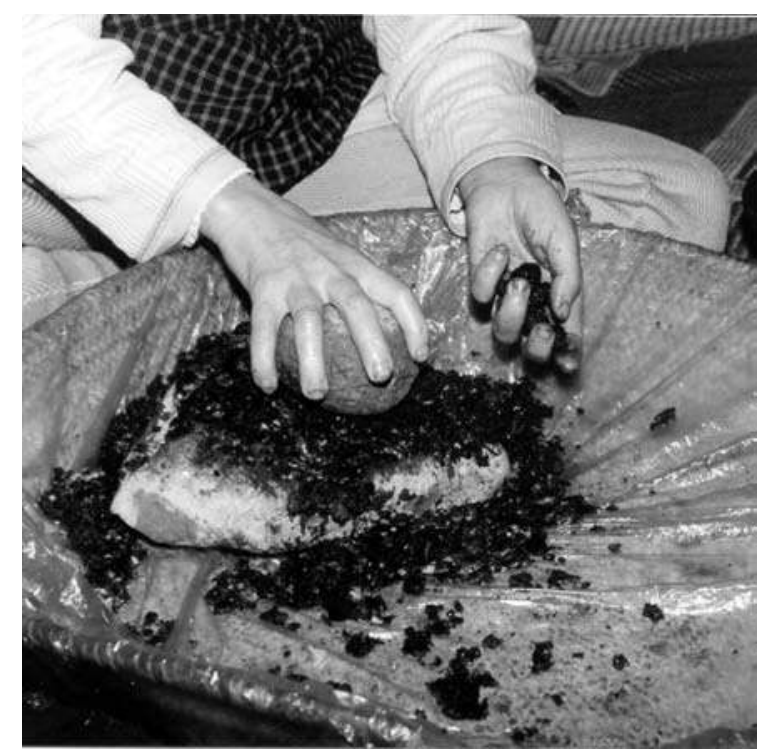

La pierre dormante, lourde, haute et plate (staha) est érigée au fond du van, tandis que la pierre arrondie (udi) ${ }^{17}$, tenue de la main droite écrase le fruit (pulpe et noyau) par percussion lancée. De cette opération, qui va durer 90 minutes, résulte une pâte grossière, constituée de pulpe et de débris de noyaux d'olive (appelée pâte d'olives).

17 Utilisée aussi par les potières pour briser les rebuts de poterie. 


\section{Le malaxage}

La pâte, chauffée sur un brasero alimenté par du charbon de bois de chêne (bllut), va être malaxée dans une jatte en argile avec une faible adjonction d'eau chaude (fig. 6). L'opération dure une quinzaine de minutes, elle sera suivie de la mise en pâte dans les scourtins (enscourtinage) et du pressurage.

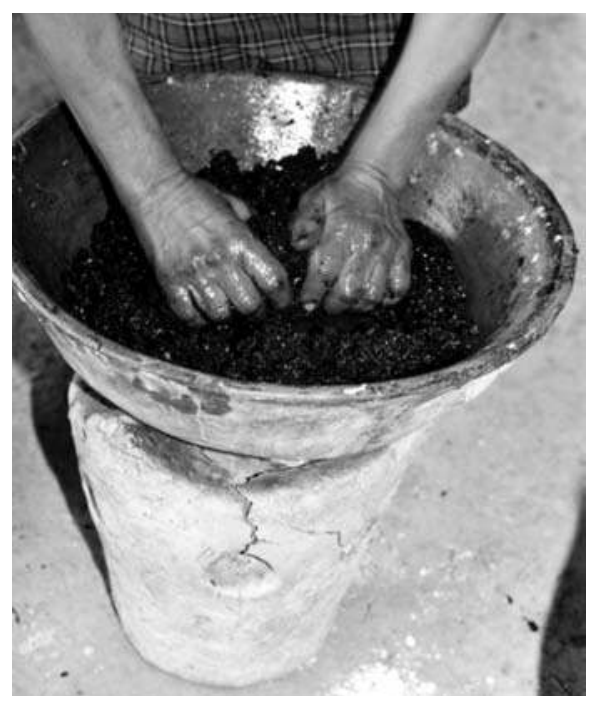

Figure 6. Le malaxage.

Le pressurage

La pâte d'olives est alors introduite dans des paniers ronds, appelés scourtins, qui laissent échapper le liquide en retenant les pulpes et les noyaux. Le choix des fibres végétales pour la fabrication des paniers, chwami, est une exigence technique des presses en bois à vis latérales ou à levier : ils doivent retenir le moins d'huile possible. Le jonc, smar (Juncus maritimus Lamk), mais surtout la feuille de palmier nain, doum, (Chamaerops humilis L.), ${ }^{18}$ présent à l'état sauvage pendant la saison des olives, sont particulièrement appréciés. Ouvertes sur la face supérieure, ces vanneries tressées sont fabriquées et vendues ${ }^{19}$ par les hommes sur les marchés de Sbt Tsoul, Zrizr, Ghiyata à Oued Amlil (Taza).

\footnotetext{
18 Au contraire des scourtins en fibre de noix de coco et de plastique, ou d'alfa, largement utilisés dans les presses hydrauliques lorsque la pâte d'olive est mécaniquement distribuée sur les scourtins.

1910 dirhams (jonc) ; 12,50 dirhams (doum).
} 
Les paniers, chargés de cette pâte (fig. 7), sont posés sur la maie du plateau inférieur de la presse. Par serrage manuel et progressif des écrous, le plateau presseur est abaissé sur les paniers (un ou deux) et se rapproche parallèlement de lautre plateau. Ce serrage induit certains avantages : possibilité de produire rapidement une huile des prémices qui va faire apprécier l'huile de l'année ; durée d'extraction rapide, pression accrue, rendement supérieur en huile (qualitatif et quantitatif), économie d'énergie humaine, autonomie de l'ouvrière. La presse, inclinée contre le mur va exercer une pression jusquà épuisement de la pâte, sans intervention humaine continue. L'huile coule lentement dans la jatte placée sous le bec. Il n’y a pas de décantation, les olives ayant perdu les margines amères dans la chaleur du four.

Figure 7. Préparation des paniers.

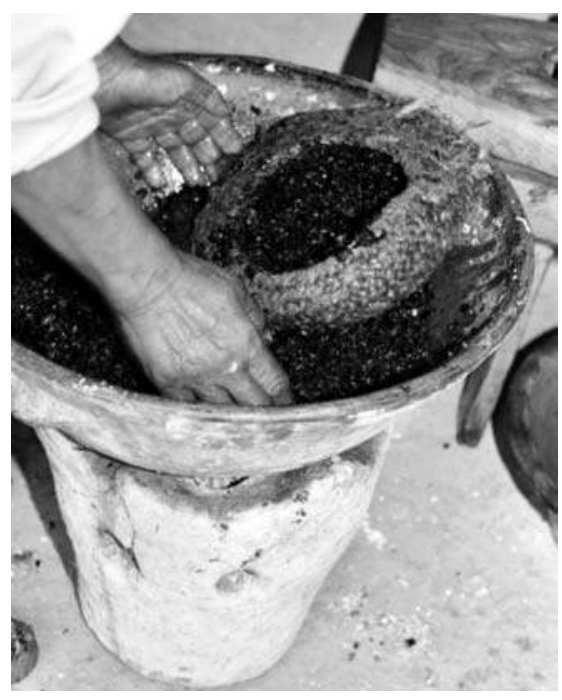

Environ 75 cl d'huile ont été extraits des 5 kilogrammes d'olives fraîches ${ }^{20}$ ce qui montre bien lintention de produire une huile gastronomique.

Au terme de cette ultime opération, la presse est lessivée à la brosse, séchée au soleil, enveloppée dans un sac, avant d'être rangée dans la cuisine ou le débarras.

2014 kg d'olives fraîches (fruits non gorgés d'eau) donnent environ 2 litres d' 'alwana. 


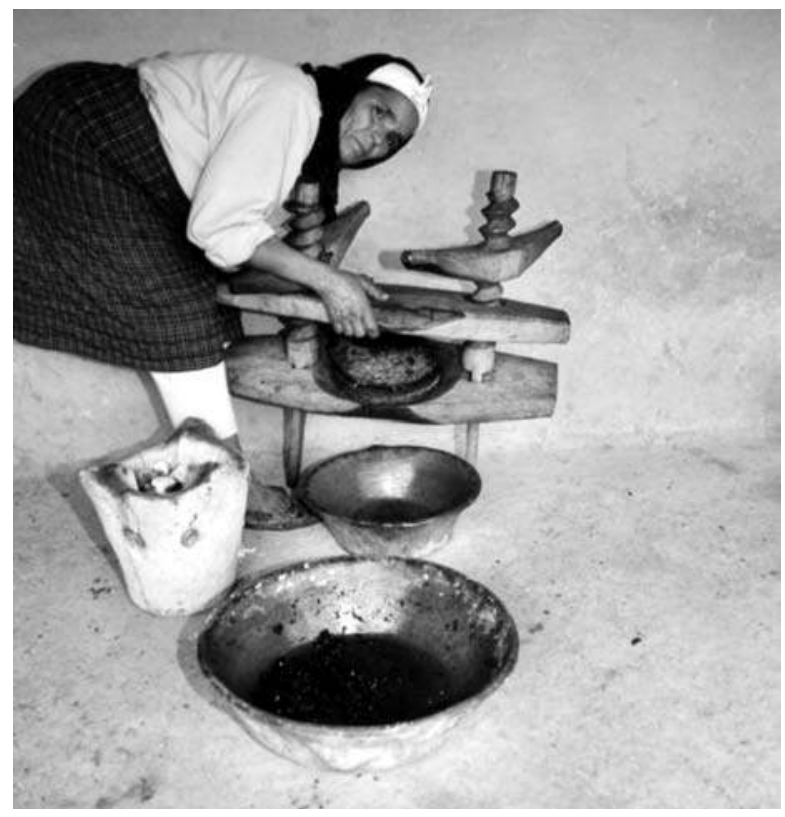

Figure 8. Le pressurage.

\section{La conservation}

La cueillette des olives coïncide avec les labours. Elle sétend, selon les années, de la mi-novembre à la mi-janvier mais la conservation des olives destinées à l'extraction peut se poursuivre jusqu'en avril, ultime moment avant leur broyage et pressurage dans l'huilerie familiale. Quant à l'huile des prémices au goût et au parfum si caractéristiques, elle est conservée dans une petite jarre (jabya ou chbriya), pour être consommée crue avec les crêpes (mlwiya et rígayf) du petit-déjeuner.

Les fruits non exprimés au cours de la cueillette sont stockés, comme à 'Ayn Lhaj 'Mr, dans un haut silo de roseau, recouvert de terre et d'argile blanche. Ce silo conserve dans le sel gemme (mlh lhiya) au pouvoir salant élevé, extrait de la mine proche de Tissa, les olives fraîches pour une extraction ultérieure de l'huile autre que l'alwana. Tel procédé de conservation est réputé éloigner la mouche des fruits Dacus oleœ : dubibat zzitun. Chaque couche d'olives est étalée sur un lit de gros sel et recouverte de gros sel. En revanche, dans le Souss (Anti Atlas central), les fruits séchés 
au soleil d'hiver sont conservés sans sel et l'huile en est extraite tout le long de l'année. Reconnue pour ses principes actifs, l'huile conservée plusieurs années (zit lhayla ou zit tryaq) est réservée à des fins médicinales (refroidissement des animaux et des humains). Quant au tourteau (lfitor), résidu de cette extraction, il sert de combustible aux fours.

\section{Autres procédés féminins d'extraction de l'huile d'olive}

L'exemple du procédé d'extraction d'alwana, caractéristique d'un savoir technique féminin et régional, ne doit pas occulter les autres procédés féminins d'extraction de l'huile. On observe à Taounate et à Ouazzane, mais aussi dans le Souss 21 , des procédés antérieurs ou concomitants au pressoir à coins (et au moulin ?) nécessitant une force musculaire et des outils rudimentaires. Cinq manières de faire illustrent ce procédé qui consiste à écraser les olives entre deux pierres et à installer la pâte obtenue dans un linge disposé sur une pierre inclinée recevant une charge de pierres superposées.

- L'olive fraîche (vert-rouge) est écrasée à la pierre, triturée et pressée à la main avec ajout d'eau chaude. L'huile émerge par décantation.

- Lolive est écrasée entre deux grosses pierres surélevées (sur une table par exemple), la pierre du bas étant inclinée pour permettre l'écoulement du liquide oléagineux dans un plat en terre cuite, placé au-dessous. Ce procédé de pression ne nécessite aucune intervention.

- L'olive verte gaulée est concassée, comme pour l'alwana, puis déposée dans une jatte. L'huile décantée est écopée à l'aide d’une étoffe de laine (mndil dial suf). La pâte restante est installée dans les paniers et abandonnée à la presse domestique.

- À Ouazzane (Zoumi), la pâte d'olives est foulée dans une cuve dallée (ssahrij) installée dans l'oliveraie, alimentée par l'eau de source ou de pluie ; l'huile décantée est recueillie par écopage à l'aide d’une étoffe de laine, appelée ici sntafa.

${ }^{21}$ Et même à Palmyre, oasis située dans la partie septentrionale du désert syrien (à $230 \mathrm{~km}$ au nord-est de Damas), où l'extraction de l'huile de térébinthe (zet mn el botem) se déroule ainsi : après broyage dans un moulin (à grains) et lavage, la pulpe de térébinthe, cueillie l'été, est longuement pétrie dans l'eau chaude, puis mise dans une bâche (makbūse) (panier ouvert fabriqué avec des feuilles de palmier cousues) et brassée. La bâche repose sur des morceaux de bois au-dessus du pressoir (al-ma'șara). Ce dernier est une pierre dans laquelle une auge est creusée pour l'écoulement de l'huile. Des pierres sont installées sur la bâche (Cantineau 1934, I : $44-45$ et dessin). 
- Dans le Souss, les olives vertes cueillies à la main sont brisées entre deux pierres puis mises à cuire à la vapeur dans un couscoussier et pressées entre les doigts. L'huile ainsi exprimée est nommée tahlwant ${ }^{22}$.

Le fait que ces procédés féminins n'ont subi aucun "perfectionnement " ne saurait être élucidé sans l'éclairage du contexte anthropologique de zones éloignées des voies de circulation. Dans le monde rural, où le rôle primordial de la femme (mère ou épouse) est de gérer rigoureusement la matière culinaire de base (ici céréales et huile d'olive), son statut, sa respectabilité et son honorabilité sont intimement liés à ce savoir technique. Pour pallier le manque de cette matera prima, le choix culturel est fait de réaliser son huile propre, dans l'indépendance de l'espace domestique et lignager qui est le sien avec les outils adaptés à son mode de vie. On remarque qu'outil rudimentaire, procédé technique et travail musculaire, mobilisent une mémoire de femmes : corporelle (position assise/courbée); technique (construction de fours, poteries); spatiale (domestique, verger); temporelle (cueillette et moisson saisonnières); économique (gestion domestique).

\section{La grande presse à vis latérales}

C'est, à première vue, la version grand format de la petite presse domestique. Outre une taille plus imposante, elle se caractérise par une fixation au sol, un plateau unique, quatre écrous, une barre de rotation, une utilisation masculine et une productivité plus importante. On la rencontre dans l'aire de pressurage, contiguë au verger ${ }^{23}$, qui rassemble le moulin en pierre, le bassin de lavage des olives et le bassin de décantation. Elle se compose de divers éléments monoxyles (chêne, noyer réputé le plus adéquat, caroubier « mâle», peuplier, micocoulier):

- deux montants (ou vis) hauts de $249 \mathrm{~cm}$ dont la base circulaire (IO $8 \mathrm{~cm}$ de hauteur) est partiellement (à $80 \mathrm{~cm}$ ) scellée dans une table de ciment et de graviers (une tige métallique traverse le milieu de chaque montant [partie scellée invisible] afin de l'immobiliser);

- un plateau horizontal percé vers ses extrémités pour le passage des vis ;

- deux paires d'écrous ;

\footnotetext{
22 Forme berbére de 'alwana

23 Dans le Souss, le pressoir est toujours à l'abri de l'Cil envieux, des intempéries et autrefois des luttes entre sédentaires et nomades.
} 
- une barre de serrage (ou de rotation) retenue par un anneau de fer autour de l'écrou. Cette barre ne traverse pas la vis comme dans la base de la vis d'une presse à levier ;

- une planche en bois arrondie, épaisse et munie d’une poignée ${ }^{24}$ quelquefois percée pour le passage d'une corde facilitant sa préhension lorsque le bois est gras. Sa fonction est de faire tampon entre la pile de paniers gras et le plateau de la presse, en assurant l'adhésion et la pression de ce dernier sur la pile ;

- une maie, cuvette surélevée bâtie au centre de la table (entre les montants), au pourtour creusé pour l'écoulement de l'huile vers le bassin de décantation.

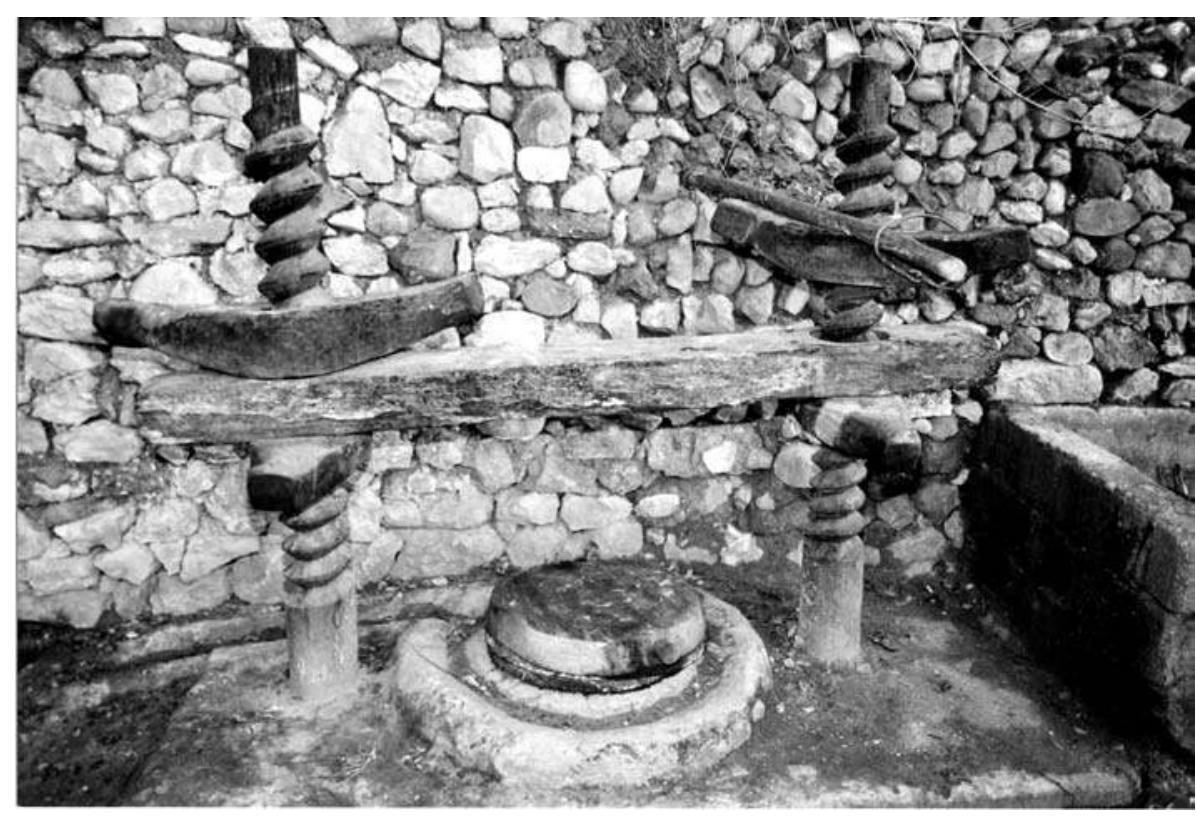

Figure 9. La grande presse à huile familiale.

La dimension des éléments de la presse familiale, la présence de la barre de serrage et de la planche, la taille et le matériau des paniers (alfa préféré au

${ }^{24}$ Ou deux. Telle planche est utilisée dans une presse à levier (vis et contrepoids) à Moulay Idriss (Bni Merghz) et dans une presse métallique à double vis. 
jonc) sont tous imposés par une quantité de pâte d'olives nettement supérieure à celle attendue de la petite presse domestique. On relève, dans la grande presse familiale, un progrès notoire : la barre de serrage n'est pas engagée dans la cavité percée dans la vis en bois mais dans un anneau engagé autour de l'écrou. De ce progrès résulte un serrage plus sûr qui évite l'éclatement de la vis.

Nous avons dit la rareté de ces presses en bois dans le verger, où les hommes conduisent la trituration des olives et le pressurage de l'huile. Après lavage et ventage (opération qui consiste à débarrasser les fruits des feuilles et des brindilles) dans le bassin de lavage, les olives sont broyées dans le moulin en pierre. La pâte obtenue est alors installée dans les paniers (8 à IO), empilés au-dessus de la maie dont l'emplacement, à équidistance des vis, c'est-à-dire au-dessous du point d'action directe du plateau, assure simultanément la stabilité de ce dernier et une pression centrée et équilibrée sur la pile de paniers. Du remplissage régulier et de l'empilement de ces paniers ( 17 à $20 \mathrm{~kg}$ de pâte, chacun), qui exigent une dextérité certaine, dépendra l'équilibre de la pile et la quantité d'huile attendue. Les écrous vont être serrés pour permettre au plateau d'exercer une action progressive sur la pile de paniers. Le liquide oléagineux coule dans la maie puis dans la rigole aménagée jusqu’à la cuvette de décantation (nnqir) pouvant contenir 250 litres d'huile. Un orifice percé dans la partie inférieure de celle-ci permet l'évacuation des margines vers l'extérieur. L'huile est récupérée par décantation. Ici, outil rudimentaire, procédé technique et effort musculaire mobilisent une mémoire masculine : corporelle (position debout), spatiale (champ, marché) ${ }^{25}$, temporelle (travaux agraires : labours, moissons, gaulages), économique (famille, commerce).

${ }^{25}$ Comme lieu d'externalisation de la production domestique, de son trans-fert. 


\begin{tabular}{|c|c|c|c|}
\hline Éléments & $\begin{array}{l}\text { Appellations } \\
\text { vernaculaires }\end{array}$ & $\begin{array}{l}\text { Essence } \\
\text { végétale }\end{array}$ & $\begin{array}{c}\text { Dimensions } \\
(\mathrm{cm})\end{array}$ \\
\hline Montant (vis, 2) & $m \dot{g} z l$ & Caroubier mâle & Hauteur : 249 \\
\hline Plateau horizontal & uṣla & Caroubier mâle & Longueur : 205 \\
\hline Écrou & hnzzira & Caroubier mâle & $86 \times 13 \times 13$ \\
\hline Barre de serrage & hrawa & $\begin{array}{l}\text { Oléastre ou } \\
\text { caroubier }\end{array}$ & $\begin{array}{l}\text { Longueur : } 107 \\
\text { Diamètre : } 5\end{array}$ \\
\hline Planche & qurșa & & Diamètre : $60-62$ \\
\hline Maie & jfna & & Diamètre : 104 \\
\hline Scourtin & $\begin{array}{l}\text { chamma } \\
\text { /chamya }\end{array}$ & Alfa & $\begin{array}{l}\text { Diamètre : } 60-66 \\
\text { Ouverture : } 40\end{array}$ \\
\hline
\end{tabular}

Tableau 2. Caractéristiques de la grande presse à vis latérales.

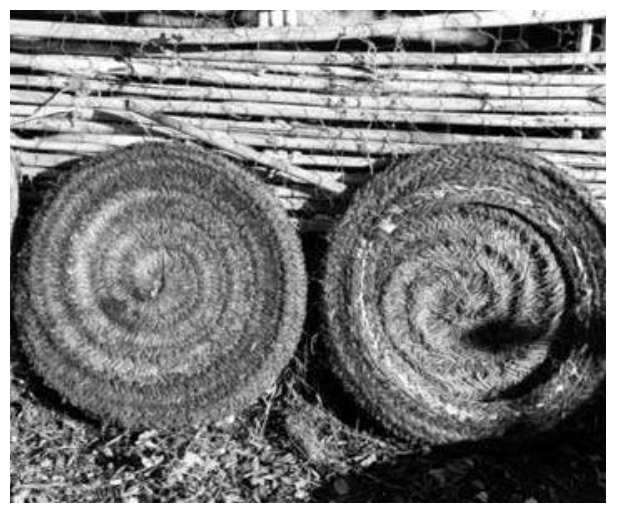

Figure 10. Scourtins. de la presse familiale.

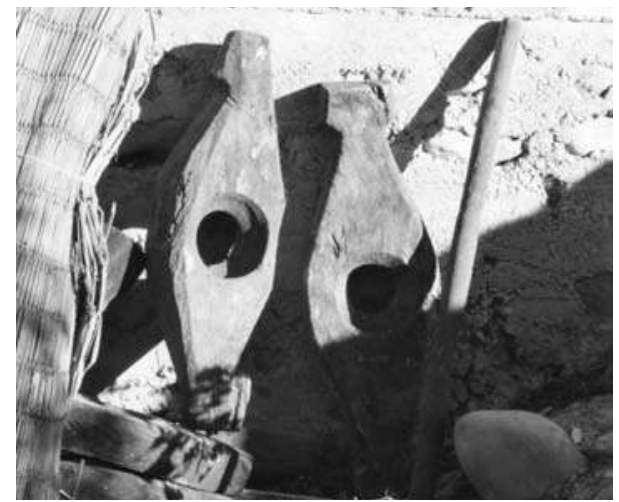

Figure 11. Ecrous et barre de serrage rangés. 


\section{Comparaison des deux presses à vis latérales}

Plusieurs caractéristiques distinguent les presses à vis latérales de Taounate : mobilité, dimension, traitement des olives et consommateurs.

La presse domestique, entièrement démontable et transportable à l'instar du métier à tisser, est de faible dimension et de poids. Le traitement particulier de l'álwana nécessite un four où par dessiccation les fruits sont débarrassés des margines. L'huile ainsi extraite est réservée au foyer.

La taille et le poids de la presse familiale imposent des vis scellées au sol dans une table de ciment (au tiers de leur hauteur totale), au contraire des autres éléments : plateau, écrous) qui sont démontés lors des intempéries. Elle est utilisée par les hommes. La trituration d'une plus grande quantité d'olives impose la force du moulin en pierre à meule unique, animé par une mule. L'huile ainsi extraite est destinée à la table familiale et à la vente (30 dirhams le litre, en 2003).

\begin{tabular}{|c|c|c|}
\hline Caractéristiques & $\begin{array}{l}\text { Presse domestique } \\
\text { féminine }\end{array}$ & $\begin{array}{l}\text { Presse familiale } \\
\text { masculine }\end{array}$ \\
\hline Description & $\begin{array}{c}\text { Mobile } \\
2 \text { plateaux } \\
2 \text { écrous } \\
2 \text { clés de blocage }\end{array}$ & $\begin{array}{c}\text { Scellée au sol (à la table) } \\
1 \text { plateau } \\
4 \text { écrous } \\
1 \text { planche } \\
1 \text { barre de rotation }\end{array}$ \\
\hline Utilisation & Intérieure (foyer) & Extérieure (verger) \\
\hline Destinataires & Table familiale & Table du maître (commercialisée) \\
\hline Liquides oléagineux & $\begin{array}{l}\text { Sans margines } \\
\text { / huile vierge }\end{array}$ & $\begin{array}{c}\text { Avec margines } \\
\text { plusieurs pressions }\end{array}$ \\
\hline Broyage & Manuel / pierres brutes & Animal / moulin en pierre \\
\hline Rendement en huile & Faible & Important \\
\hline Consommation de l'huile & Immédiate, crue & Conservation, crue/cuite \\
\hline
\end{tabular}

Tableau 3. Comparaison des deux presses à vis latérales.

D'autres questions se posent. On admet que pour extraire l'huile, laction du moulin précède celle de la presse. On peut done se demander pourquoi de 
nombreuses huileries (coopératives ou privées) conservent l'usage mixte du moulin en pierre (actionné par un animal) et de la presse métallique (électrique/hydraulique) ? La réponse serait de nature économique mais pas seulement: proximité d'une carrière meulière ; prix prohibitif du moulin métallique, quantité restreinte d'olives à triturer, présence d'animaux dans lagriculture et le transport, affect des producteurs-consommateurs pour une huile dont les caractéristiques organoleptiques répondent au type d'outils. Inversement, on note que le couple moulin métallique / presse en bois (à levier) est inconnu. On peut bien sûr y déceler le signe d'une rationalité technique, par degrés, reflet d'une réalité économique car finalement ce sont les huileries familiales de montagne (Taounate, Moulay Idriss-Zerhoun, Anti-Atlas), d’accès difficile, qui conservent l'usage couplé du moulin en pierre (transporté par roulage des meules) et de la presse en bois.

Lintroduction de l'électricité modifie l'équipement de l'huilerie, quand elle ne signe pas son adaptation à des besoins nouveaux. Déjà, l'équipement des unités modernes de trituration en chaine à système continu ${ }^{26}$ d'extraction de l'huile et de sa mise en bouteille se propagent aux abords des routes chez les entrepreneurs nantis.

L'extension des zones oléicoles, liée à l'accroissement de la plantation d'oliviers ${ }^{27}$ et des récoltes; l'amélioration de la qualité des olives et de l'huile signent un nouvel essor de la productivité. Ce progrès concomitant à l'emprunt d'une seule machine très performante s'étend, malgré son prix très élevé, pour satisfaire une demande croissante d'huile d'olive dans le monde. Cette machine ${ }^{28}$, nécessitant deux ouvriers placés chacun à une extrémité de la chaîne, est capable d'effeuiller, laver et triturer environ 6o tonnes d'olives par jour, voire davantage. Rappelons, qu'à l'instar de l'huile d'argane, ce traitement fait perdre à l'huile d'olive les qualités organoleptiques de terroir.

\footnotetext{
${ }^{26}$ La première de ces machines a été offerte à l'Institut Technique Agricole de Sahel Boutaher par le Conseil Oléicole International (COI) en 2001. Cet ITA dispose d'un laboratoire d'analyse des huiles et des olives.

27 Le plan national de développement oléicole (1998-2010) prévoit d'intensifier les superficies à planter à l'horizon 2010, estimées à 550000 ha en 2001 (Source : Maroc-ministère de l'Agriculture, du Développement Rural et des Eaux et Forêts (MADREF / Campagne 2000/2001).

28 Composée de plusieurs unités : 1 - bassin-récepteur d'olive ; 2 - laveuse-effeuilleuse ; 3 - broyeur ; 4 distributeur de pâte d'olive ; 5 - malaxeur ; 6 - presse ; 7 - séparateur liquide/solide ; 8 - séparateur liquide / liquide ; 9 - caméra-tube propulsant les tourteaux et les margines. Dans cet espace, les grignons servent de combustibles pour chauffer l'eau adjointe à la pâte d'olive.
} 
Les presses en bois à vis latérales, actives dans leur contexte culturel, éclairent le lien entre le mode d'existence des agriculteurs (autonomie, sinon «autarcie»), les choix culturels et techniques, l'affect et le goût des producteurs. Ces presses doivent leur pérennité à un ensemble de facteurs, qualitatifs d'abord, soumis au respect du mode d'existence des acteurs, qui a imposé leur maintien. Ainsi, le résultat attendu de ce type de presses est atteint par les réalisateurs-consommateurs, nés dans ce paysage oléicole ancien ${ }^{29}$. Il expliciterait l'indifférence, voire l'abandon de la presse à treuil (concomitante à la presse étudiée ici) connue dans la proche Volubilis, mais aussi de la presse à levier en voie de disparition. Fait prégnant, la presse à vis latérales en métal supplante largement la presse familiale en bois dans l'espace rural. Ce modèle, plus résistant, coïncide avec lintention, non seulement de produire davantage d'huile d'olive mais de s'affranchir des aléas du bois, matériau qui exige une plus grande attention.

Outre la description de la presse en bois à deux vis latérales donnée par Héron et transmise par Qustā ibn Lūqā (Liban), il existe quelques indices techniques non développés ici, mais qui pourtant mériteraient d’être retenus. Ces indices suggèrent une parenté technique avec le Proche-Orient et font progresser notre interrogation sur la présence de la presse à double vis à Taounate: la fabrication domestique de l'huile de térébinthe à Palmyre (Syrie), le procédé persan de fabrication de l'huile à partir d'olives noires séchées dans un four (l'Iran ne connaît pas la presse à vis), la désignation actuelle des scourtins à Taounate (chamya ou chamma, lit. celle du pays de Cham : Syrie-Palestine) et enfin la presse signalée en Crête au XI $\mathrm{XI}^{\mathrm{e}}$ siècle ${ }^{30}$ ou celle connue en Provence ${ }^{31}$ jusqu'à la seconde moitié du XVII siècle. Tous ces témoins civilisationnels invitent à réfléchir sur linvention et l'emprunt, mais surtout sur l'utilisation de telles presses (la vis est méditerranéenne), notamment leur diffusion sur les rives de la Méditerranée, particulièrement entre le Maghreb et le Machreq. Ils montrent, en tout cas, lintérêt de

\footnotetext{
29 Des résultats de fouilles et d'analyses de charbons de bois, datés du courant du IX millénaire $\mathrm{BC}$, ont révélé un échantillon d'Olea europæe (oléastre ?) découvert dans la grotte des Pigeons à Tafoughalt, au Maroc oriental (Roche 1958-59) au nord-est de mon site d'observation.

${ }^{30}$ Une presse quasi analogue aurait été connue en Crête, île dominée par les Arabes au XI siècle, dont seul le trapetum en pierre monolithe a été retrouvé dans la campagne (information due à M. Ch. Vallianos, directeur du musée d'ethnologie crétoise, Vori). Cf. Parharides, Ioannou \& Vallianos 2001. Il n'existe malheureusement aucun autre témoin matériel de sa présence, le matériau végétal n'ayant pas résisté au temps.

31 Il s'agit de presses à vis centrale (unique) mentionnées par A. Casanova (1978). Cf. Delmas 1988.
} 
développer ce lien à peine suggéré, qui enrichirait la connaissance de cette presse, vestige archaïque ou invention adaptée maintenue jusqu’à nos jours.

On n'oublie pas qu’au Maroc même, une ligne de séparation technique d'extraction de l'huile entre le Nord et le Sud interpelle. La géographie des matières oléagineuses, Nord méditerranéen : oliviers / Sud africain : arganiers, induirait-elle une frontière de l'outillage et des procédés pour extraire l'huile alimentaire, sachant que l'outil est adapté au geste et aux habitudes musculaires? L'histoire et la mémoire des hommes (corps, outils, procédés), comme celles des plantes (sauvages ou cultivées) signent en quelque sorte l'évolution ou la stabilité technique si aucun fait majeur ne vient modifier cet état. De même, on se souviendra que les presses ont servi à extraire des liquides oléagineux et non oléagineux et qu'elles ont été utilisées ici et là comme réponses à une nécessité.

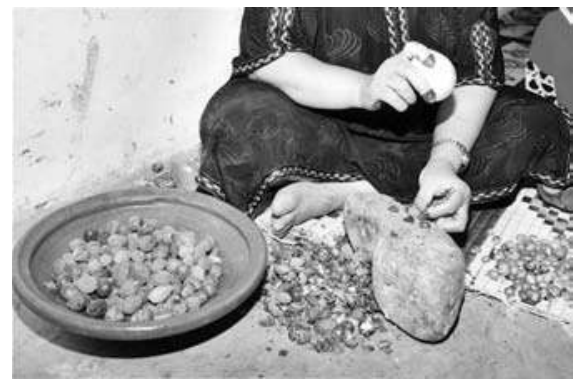

Figure 12. Dépulpage de noix d'argane

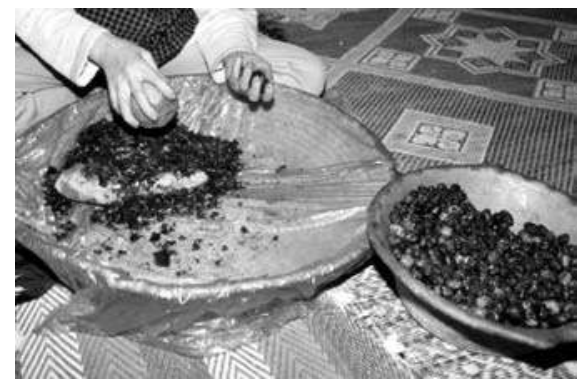

Figure 13. Concassage des olives.

Nous voudrions clore cette note par une analogie technique propre à un autre procédé féminin millénaire d'extraction de l'huile, que nous avons eu l'occasion d'observer au Maroc. Qu'il s'agisse de dépulper et d'écaler le fruit dur de l'arganier (Argania spinosa L.) ou de concasser le fruit de l'olivier (Olea europea L.), les procédés sont en tous points similaires dans leur mise en ouvre d'objets naturels non transformés par la main de l'homme, de leur mode d'action par percussion lancée mais aussi dans la position du corps des ouvrières pendant cette activité, dont l'intention de produire du lien social à travers une économie domestique prioritaire est commune. 


\section{Références}

Amouretti, Marie-Claire, Georges Comet, Claude Ney \& Jean-Louis Paillet 1984 «À propos du pressoir à huile : de l'archéologie industrielle à l'histoire ", Mélanges de l'École Française de Rome 96 : 379-42I.

Caillot, Olivier

I984 Huileries antiques de Syrie du Nord. Paris : P. Geuthner

Cantineau, Jean

1934 Le Dialecte arabe de Palmyre, I: Grammaire; II : Vocabulaire et Textes. Beyrouth : Institut français de Damas.

Carra de Vaux, Bernard

I893 Les Mécaniques ou l'élévateur de Héron d'Alexandrie. Traduction accompagnée de la version arabe de Costa ibn Lūqa. Journal asiatique 9 (I-2) (rééd.I988, Paris : Les Belles-Lettres).

Casanova, Antoine

1978 Techniques. Société rurale et idéologie en France à la fin du XVIII siècle. Paris : Les Belles-Lettres, 1978 : 19-20

Cresswell, Robert

I996 Prométhée ou Pandore? À Propos de technologie culturelle. Paris : Kimé.

Delmas Jean

I988 Pressoirs et presses rouergats. Rodez: Musée du Rouergue (Guide des Arts et Métiers $n^{\circ}$ I2).

El Alaoui, Narjys

I999 «Paysages, usages et voyages d'Argania spinosa (L.) Skeels $\left(\mathrm{XI}^{\mathrm{e}}-\mathrm{XX}^{\mathrm{e}}\right.$ siècles) ", Jatba 4I (2) : 45-80.

$200 \mathrm{I}$ "L'olivier, l'huile et les rites en Idaw Martini - Sud du Maroc ", in

L'Olivier dans l'espace et dans le temps. Les relations d'un arbre avec son terroir. Actes des I ${ }^{\text {eres }}$ Rencontres Internationales de l'Olivier,

Nyons : Institut du Monde de l'Olivier : $38-42$.

2002 "Le Maroc", in L'Huile d'olive I. De la culture traditionnelle au symbole alimentaire mondialisé. Musée national des Arts et Traditions populaires (Rapport d'enquêtes) : 93- II7.

2003 "Meules et moulins du Sud marocain ", in Meules à grains. Actes du colloque international de la Ferté-sous-Jouarre. Paris : Maison des Sciences de l'Homme-Ibis Press : 51-60.

Mohebbi, Parviz

I996 Techniques et Ressources en Iran du VII au XIX siècle. Téhéran : Institut français de recherche en Iran (Bibliothèque iranienne 46 ). 
Parain, Charles

I979 «Typologie des pressoirs préindustriels et aires de diffusion des types successifs en Europe occidentale ", in Outils, ethnies et développement historique. Paris, Éditions Sociales (Terrain).

Parharides, S., C. Ioannou \& Christian Vallianos

200I Ancient Technology at the Time of Heron of Alexandria and its Consequent Decline and Simplification : an Example Drawn from the Twin-Screw Press in Extraordinary Machines and Structures in Antiquity. University of Patras and The Society of Ancient Greek Technology (International Symposium).

Roche, Jean

I958-59 "L’Épipaléolithique marocain ", Libyca VI- VII : I59-I92.

Trochet, Jean-René

1993 Aux origines de la France rurale. Outils, pays et paysages. Paris, : Éditions du CNRS (Mémoires et documents de géographie).

Vignet-Zunz, Jacques-Jawhar

2002 "Roues, bielles, pistons dans le Rif occidental (Maroc) ", Cahier

d'Histoire des Techniques V : Agriculture méditerranéenne. Variété des techniques anciennes: $247-259$. 


\section{Annexe 1. Glossaire arabe-français}

\begin{tabular}{|c|c|}
\hline brbuch & tenon (oreille) de jatte, pl. brabch \\
\hline chamya / chamma & $\begin{array}{l}\text { panier rond en fibres végétales tressées contenant la pâte } \\
\text { d'olive pendant l'extraction de l'huile; pl. chwamy }\end{array}$ \\
\hline chih & contrepoids en pierre d'une presse à levier \\
\hline dum & $\begin{array}{l}\text { palmier nain (Chamaerops humilis L.) utilisé pour la } \\
\text { fabrication des scourtins }\end{array}$ \\
\hline fitur / lfitor & $\begin{array}{l}\text { tourteaux (résidus secs de la pâte d’olive) aux usages } \\
\text { variés: combustion, fabrication du savon noir, matériau } \\
\text { de construction }\end{array}$ \\
\hline frna & $\begin{array}{l}\text { four en ogive construit par les femmes (cuisson du pain, } \\
\text { déshydratation des olives) }\end{array}$ \\
\hline frur & $\begin{array}{l}\text { débris ou rebuts de poterie concassés pour servir de } \\
\text { matériau de construction à l'assise du four à pain }\end{array}$ \\
\hline$g s^{\prime} a$ & jatte en argile rouge \\
\hline hlbu / hlib zitun & cueillette manuelle des olives $\rightarrow$ tsram \\
\hline hrawa & barre de serrage ou de rotation (presse familiale) \\
\hline hnzira & écrou ; pl. hnzzirat ou hnnazr \\
\hline jfna & cuvette ou maie creusée dans la table (presse familiale) \\
\hline lhawḍ / lbaṣt & plateau inférieur (presse domestique) \\
\hline lm'gun / zrrab & $\begin{array}{l}\text { bec de plateau inférieur (presse domestique) } \\
\rightarrow \text { lhawd/lbaṣt }\end{array}$ \\
\hline lm‘șra & presse à huile, huilerie, pressoir, aire de pressurage \\
\hline lm‘șriya & petite presse à huile à deux vis, à usage domestique \\
\hline luha / hnzira & plateau mobile d'une presse domestique \\
\hline
\end{tabular}




\begin{tabular}{|c|c|}
\hline lyed & poignée du plateau (presse domestique) \\
\hline$m \dot{g} z l / l m \dot{g} z l$ & vis (presse domestique ou familiale); pl. $m \dot{g} a z l$ \\
\hline mida & maie (cuvette) de presse domestique \\
\hline miduna & large van spiralé utilisé pour le concassage des fruits \\
\hline mjmr & $\begin{array}{l}\text { brasero pour réchauffer la pâte d'olive avant son } \\
\text { pressurage }\end{array}$ \\
\hline na'ora & moulin hydraulique à palettes, noria \\
\hline nnadr & silo à grain ou à paille \\
\hline nuala & maison en pisé \\
\hline$q u q n a(t)$ & $\begin{array}{l}\text { écrou (presse domestique ou familiale); pl. quqnat } \\
\rightarrow \text { hnzira }\end{array}$ \\
\hline qurșa & $\begin{array}{l}\text { pièce ronde en bois, épaisse, munie d’une poignée, } \\
\text { installée au-dessus des scourtins, entre la pile de paniers et } \\
\text { la plateau mobile d'une presse familiale }\end{array}$ \\
\hline rḥa & moulin à bras en pierre \\
\hline$r h i$ & pierre meulière \\
\hline slla & haut silo à olives en roseau tressé ; pl. sllat \\
\hline tsram & cueillette manuelle des olives $\rightarrow$ ḥlbu \\
\hline$t^{\prime} m$ & $\begin{array}{l}\text { (lit. nourriture) pâte d'olive composée de pulpe grasse et } \\
\text { de débris de noyaux avant pressurage }\end{array}$ \\
\hline uṣla & plateau supérieur, mobile (presse familiale) \\
\hline
\end{tabular}




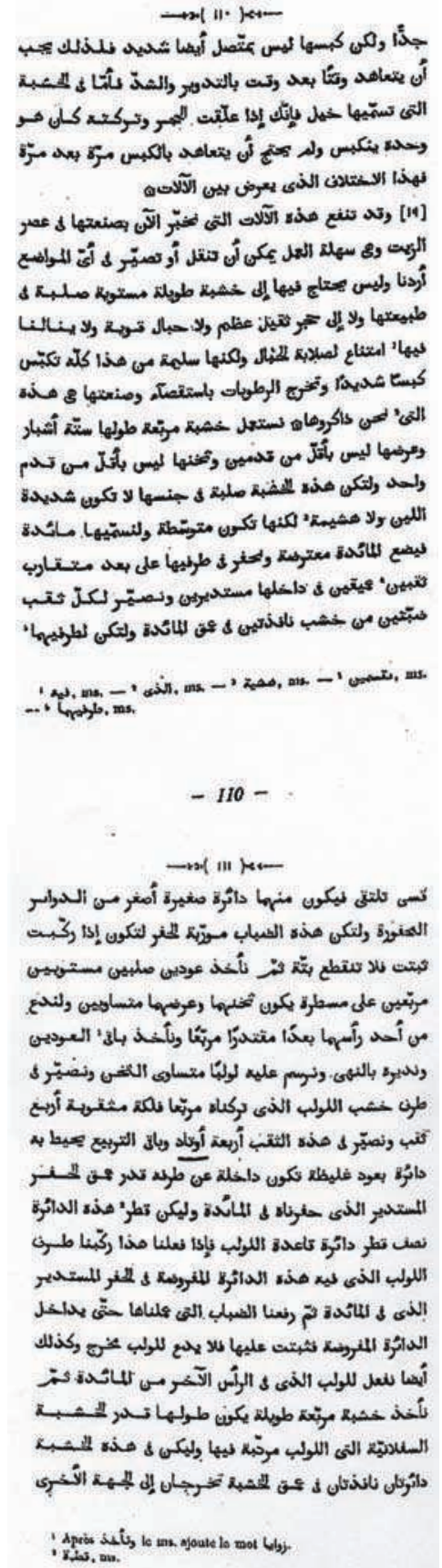

\section{Annexe 2. Extrait}

Héron d'Alexandrie, Les Mécaniques, traduction de Bernard Carra de Vaux accompagnée de la version arabe de Costa ibn Lūqa. Journal asiatique 9 (1-2), 1893, Livre III, ch. 2, paragr. 19 : 187-207.

"Ceux [les instruments $]^{1}$ dont nous allons maintenant donner la description servent à presser les olives ; ils sont dune construction aisée, et on peut les transporter et les installer partout où l'on veut. Ils ne nécessitent pas de longue pièce de bois égale dans toutes ses parties et d'une essence dure, ni de lourde et grande pierre, ni de câbles forts ; et ils ne nous offrent pas de difficulté provenant de la rigidité des cordes ; ils sont libres de tous ces inconvénients ; ils pressent d'ailleurs avec beaucoup de force et ils expriment entièrement les sucs. Leur construction est celle que nous expliquerons à lỉnstant.

Nous prenons une poutre équarrie [hasba' murabba 'a'] dont la longueur est de 6 spithames [satta' achbär], dont la largeur n'est pas moindre de 2 pieds [qadamin], et dont l'épaisseur n'est pas moindre de I pied [qadam wähid]. Cette pièce de bois doit être [I88/208] d'une essence ferme ; il ne la faut pas trop tendre ni trop sèche, mais on doit la choisir entre ces états extrêmes ; nous l'appelons la table [mā'ida']. Nous la plaçons horizontalement, et nous y creusons, non loin des deux extrémités, deux trous profonds et arrondis [taqabayn 'amîqîn]; dans chaque trou nous mettons deux loquets [dabatayn] en bois, qui, d'un côté, s'enfoncent dans l'épaisseur de la table et, de lautre côté, se terminent en demi demi-cercle; en se rencontrant, ils forment ensemble un cercle plus petit que les trous creusés. Ces loquets $[$ dabātun $]$ ont les faces obliques pour quỉls tiennent, une fois montés, sans pouvoir être arrachés. Nous prenons ensuite deux pièces de bois dur, partout égales et équarries à la manière d'une règle, leur épaisseur étant égale à leur largeur ; à l'une de leurs extrémités une longueur convenable reste simplement équarries, prenant alors par ce bout les deux pièces de bois, nous les faisons tourner, et nous traçons sur tout le reste de leur longueur une vis [lawlab] d'épaisseur constante. À l'extrémité du bois de la vis, que nous avons laissée équarrie, nous plaçons un tambour [falaka $]$ percé de quatre trous dans lesquels nous introduisons des pieux de bois $\lceil a w t \bar{a} d$, et ce qui reste de ce bout carré est revêtu d'une coiffe cylindrique en bois, ayant en tout une longueur égale à la profondeur du trou circulaire pratiqué dans la table ; une rainure circulaire $[a l$ dāira al-mafrūda'] est creusée dans ce cylindre [dāira] en bois, dont le diamètre égale la moitié du diamètre du cercle $[$ dãira $]$ de base de la vis. Cela fait, nous introduisons cette tête qui termine la vis dans le

${ }^{1}$ Les précisions et traductions entre crochets sont de l'auteur du présent article. 
trou cylindrique de la table. Nous repoussons les loquets qui ont été construits antérieurement, en les faisant entrer dans la rainure circulaire ; nous les fixons dans cette rainure, et ils ne permettent plus à la vis de sortir.

Nous faisons de même pour la vis qui est à l'autre extrémité de la table. Après cela, nous prenons une poutre équarrie et longue dont la longueur est la même que celle de la poutre inférieure dans laquelle les vis sont montées. Cette poutre est forée de deux trous cylindriques qui pénètrent dans son épaisseur et qui ressortent de lautre côté, correspondant aux deux trous cylindriques dans lesquels se place l'extrémité des vis. À l’intérieur de ces deux trous est sculptée une rainure hélicoïdale, qui fait d'eux les écrous des deux vis, en sorte que cette poutre s'abaisse lorsqu'on tourne les deux vis, et quïnversement elle s'élève lorsqu'on les tourne en sens contraire. Nous expliquerons plus loin la manière de sculpter la rainure hélicoïdale de l'écrou. La longueur et l'épaisseur de cette poutre [hachba'] doivent, comme nous l'avons dit, se mesurer à la longueur et à l'épaisseur de la table ; mais sa largeur doit être inférieure d’un quart à celle de cet organe

Nous plaçons ensuite sous la table un socle rectangulaire ayant en bas la forme d'un degré, et dont la longueur dépasse celle de la table d'une petite quantité, pour que tout l'appareil puisse être solidement dressé sur lui. Il convient de pratiquer sur une moitié du socle une entaille de dimension movenne et d'en faire une autre dans la table, de même mesure que celle qui est faite dans le pied ; puis on monte le saillant dans le rentrant, et l'appareil se trouve solidement établi. Nous installons sur la table, entre les deux vis, quatre parois [hițān] bien jointes, formées de planches minces, ayant moins d'un doigt d'épaisseur. La longueur et la largeur de l'espace carré qui se trouve entre ces planches sont telles que, la galéagre $[a l-\dot{g} a \bar{l} \bar{a}$ a $\dot{g} r a \bar{a}]$ étant placée dans cet espace, il reste autour d'elle un vide où les sucs $[$ rutūbāt $]$ puissent se répandre. Nous devons, dans le milieu de la table, pratiquer une cavité [hafran yus'a] qui ait les mêmes dimensions que la face de la galéagre reposant sur la table, afin d'entrer la galéagre dans ce creux. Nous ly établissons donc, et dans le haut, nous plaçons une planche épaisse qui occupe l'espace restant au-dessus des matières à presser ; nous la surmontons d'un chapeau [qurmiya'] moins long et moins large que la planche, dont l'épaisseur achève de remplir la galéagre. Nous tournons alors les deux vis avec les pieux qui sont dans les tambours, en sorte que la poutre formant écrou sabaisse sur le chapeau ; le chapeau et la planche qui est à lintérieur de la galéagre se trouvent refoulés ; la matière contenue dans l'appareil est pressée, et les sucs coulent. Après quoi, l’on tourne les vis dans l'autre sens, la poutre s’élève ; on ôte le chapeau, et lon renouvelle la matière soumise à la pression jusqu'à ce qu'on ait extrait tout le suc. Il existe un autre instrument à une seule vis $[. .$.$] Il y a encore beaucoup d'autres genres de presses$ [...] leur usage est très répandu et elles sont connues de tous ; elles sont d'ailleurs inférieures à celles que nous avons citées. "
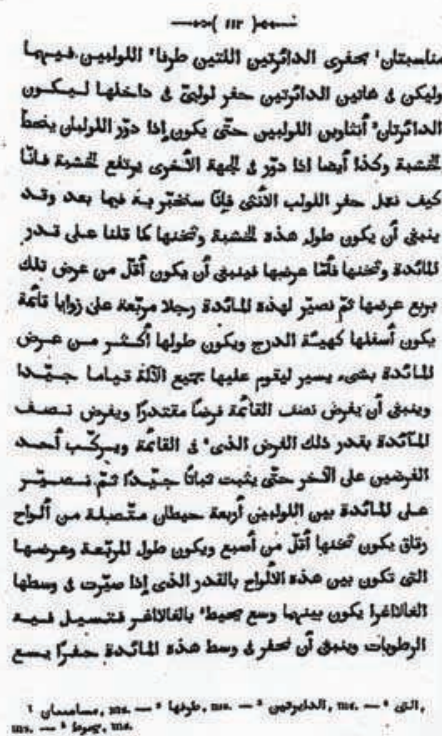$$
-112-
$$

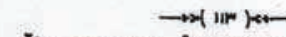

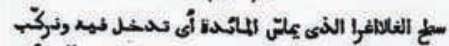

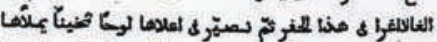

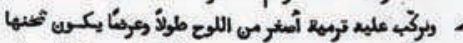

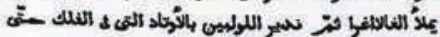

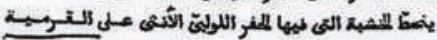

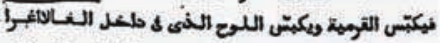

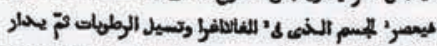

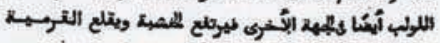

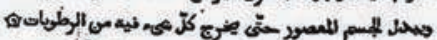
Ł

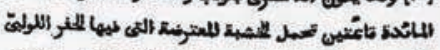

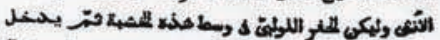

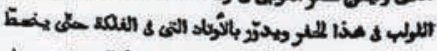

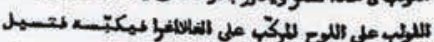

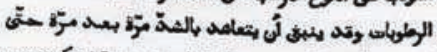

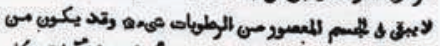

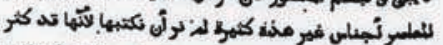

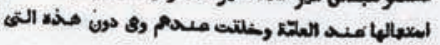
Diditis sos

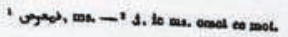
$-113-$ 